\title{
Research and Design on Vision System of the Mobile Robot
}

\author{
Xin Ma,Rongguang Sun,Yongfeng Dong \\ Underwater Acoustics Center, Navy Submarine Academy, Qingdao, 266042,China \\ E-mail: mx0537@tom.com
}

\begin{abstract}
The paper presents the design of vision system of the mobile robot, and shows methods of object recognition based on color images in the system of mobile robot. To adapt to the different light conditions, HSI color space is used. The system is able to meet the demand on rapidity and veracity of system of mobile robot. Experiment results show that the proposed technique is liable to accomplish object recognition in presence of changing illumination environment conditions.
\end{abstract}

Keywords- Mobile Robot; Color Vision; Image Processing; Object Recognition

\section{INTRODUCTION}

Autonomous mobile robot is a collection of environmental perception, dynamic decision making and planning, behavior control and implementation and other functions into an integrated system.In this article the robot uses is autonomous wheeled mobile manipulator system platform, composited mainly by the mobile platform system, five Degree of freedom manipulator system, visual system, the main control system and remote monitoring system .Vision system is the whole robot's eyes, is the main way to get external information, is the basis of the robot system. It acquisits field image continuously, proces image, analysis, complete the target identification and localization of target, and transfer the target information to the decision system.Therefore, real time performance and robustness and accuracy requirements are higher.

Robot in a closed area, use a visual sensor combining platform motion search who has the target object has color characteristics of, independent planning path movement placed target objects close to the table,and the control five manipulator grab objects.So, based on the color image segmentation in target recognition is an executable way. Have proposed color image segmentation method including clustering method, the threshold value method and the game theory with mark, regional growth and regional division merge and edge detection and so on [1]. But real time limits the application of complex visual algorithm, at the same time, visual system also should be able to adapt to the change of illumination conditions. For the system can real-time identificate the specific color target,this article use a simple and practical algorithm of double threshold image segmentation method.

\section{THE VISION SYSTEM OF HARDWARE}

Robot visual hardware mainly includes: body head installed a two degrees of freedom CCD camera, mechanical hand installed a miniature camera and research China company NANO - 7270 type embedded computer and DaHeng company DH - VT121 duplex image acquisition card.DH - VT121 video acquisition card is based on PC104 PLUS development of color/black and white video image acquisition card, just and visual computer PC / 104 PLUS slot (PCI specification) is linked together, not only low power consumption, quick speed, and have the advantages of small volume.They formed a high-speed image acquisition and processing system,is the core component of the robot vision.

\section{COLOR SPACE CONVERSION}

RGB space model is the most commonly used color space model.RGB model is based on the principle of visible light, and $\mathrm{R}$ red, $\mathrm{G}$ on behalf of the green, $\mathrm{B}$ is for blue.According to the light synthesis principle different colors of colored light mix produce another kind of color of light.And the R, G, B these three kinds of most basic color with different strength mixed can produce all color the human eye can see, so the RGB model also called additive color mode.Although facing hardware RGB color space to real-time application is very attractive, but from color invariant point of view it exists shortcomings, in three color component contains a certain brightness information of wavelength light source, light source brightness changes also affect three primary component.Therefore, it is not the most suitable for image segmentation of a kind of color space.So usually choose this can derive luminance component color space.

HSI space model is nonlinear color system.HSI system $\mathrm{H}$ is tonal (hue), used to describe color attributes; $\mathrm{S}$ is saturation (saturation), namely pure color degree of measurement; I was brightness (Intensity), and light radiation power related, said some color of light caused by the human eye visual strength. What color tonal component said is (such as red, green, blue, etc.), saturation component said color shade degree (such as deep red), luminance component descript color light and dark program (such as bright yellow, dark yellow).HSI space can better report to the color perception and identification ability, very suitable for based on color image similarity comparison.HSI model representation method is similiary with color perception of people, and in HSI space,people's perception of color is more homogeneous and therefore HSI model is fit for human visual characteristic color space, color image in HSI space, useful for the image processing, such as edge detection, image segmentation and target recognition, etc. 
The image file which CCD camera get by image acquisition card to get in memory based on pixel three primary colors RGB value stored, and by the above analysis, we can conclude that for a given color, it is difficult to extract characteristic from the RGB value.In order to improve the recognition effect so as to obtain a relatively robust color features, this paper adopted the HSI model, and carries on the RGB model to HSI model transformation.

RGB color model to HSI color model conversion formula is as follows:

$$
\begin{gathered}
H=\left\{\begin{array}{cc}
\cos ^{-1}\left(\frac{(R-G)+(R-B)}{2 \sqrt{(R-G)^{2}+(R-B)(G-B)}}\right) & B \leq G \\
2 \pi-\cos ^{-1}\left(\frac{(R-G)+(R-B)}{2 \sqrt{(R-G)^{2}+(R-B)(G-B)}}\right) & B \succ G
\end{array}\right. \\
S=1-\left(\frac{3}{R+B+G}\right) \min (R, G, B) \\
I=\frac{R+B+G}{3}
\end{gathered}
$$

\section{REAL-TIME COLOR SEGMENTATION AND TARGET} RECOGNITION

After the image acquisition,it need further image processing, including color segmentation and target recognition, etc.The whole process of image processing as shown in figure 1.

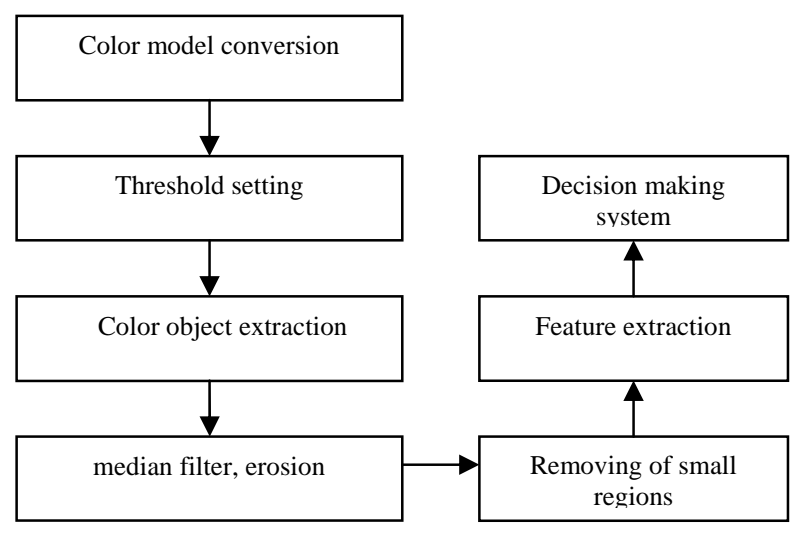

Figure 1. Image processing program diagram

\section{A. Target image extraction}

In order to improve the recognition effect so as to obtain a relatively robust color features, this paper adopted the HSI model,Three component, I was greatly influenced by the light of the component, so when use color characteristics identificate goal, to reduce brightness characteristics of I right value, mainly $\mathrm{H}$ and $\mathrm{S}$ as the main characteristics of the decision, which can improve the color feature recognition robustness.The target of a single color, and with the surrounding scenery color is unsimilar, for simplified processing, can only use chromaticity $\mathrm{H}$ target detection. The advantage of this method is not need to pixel three component in complex processing, and to reflect the difference between the main color of chromaticity $\mathrm{H}$ for processing and judgment, so that greatly shorten the color image processing time, therefore it suitable for real-time identification control occasions.In the experiment select the "H", $\quad$ "S". $H_{\min }<H<H_{\max } \quad, \mathrm{S}_{\min }<\mathrm{S}<\mathrm{S}_{\max }$, Regional image respectively tonal minimum threshold and maximum threshold value.Regional image saturation minimum threshold and maximum threshold value.Figure 2 is the yellow table tennis identification results.

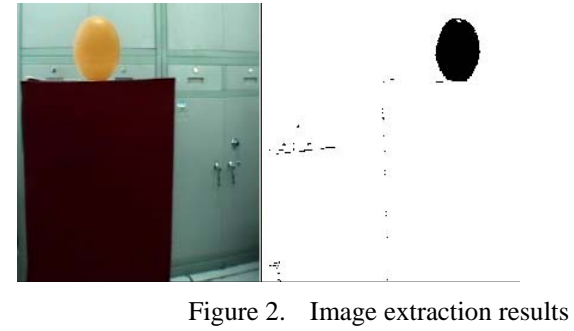

\section{B. Median filtering}

The image acquisited by CCD camera image acquisition card, because of the environment light and sensor etc interference there are noise.On the other hand, due to the image segmentation itself is susceptible to the influence of noise,So before further treatment is generally adopted some pretreatment.The traditional denoising method is to use appropriate smooth operator first for whole image smoothing, the result is often in the smooth remove noise at the same time, also make the edge of the original image become fuzzy.Median filter is a kind of nonlinear signal processing method, its purpose is to protect the image edge at the same time to remove noise.In the actual system, the twodimensional median filtering window shape and size of the effect of filter design greatly influenced.As experience speaking, for the graded a long contour line of the object image, the square or circular window advisable.Figure 3 for median filtering before and after treatment of the comparison results.

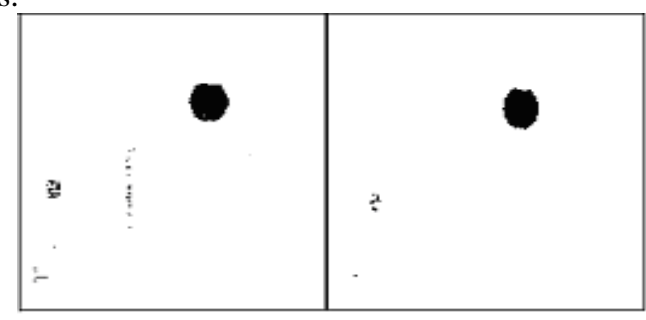

Figure 3. Median filter and image comparison

\section{Image corrosion}

Corrosion is mathematical morphology of two kinds of the most basic operation of [4], corrosion in the role of mathematical morphology is to eliminate the object boundary point, make the boundary to internal contraction process, can be less than the structural elements of the object to remove.So choosing different size structure elements, can 
remove the size of different objects. If two objects have small between the connected, through the corrosion can be divided two objects.In order to extract the object, here after a corrosion to prevent between two objects have tiny connected, for the next step of the minimum area to eliminate ready.

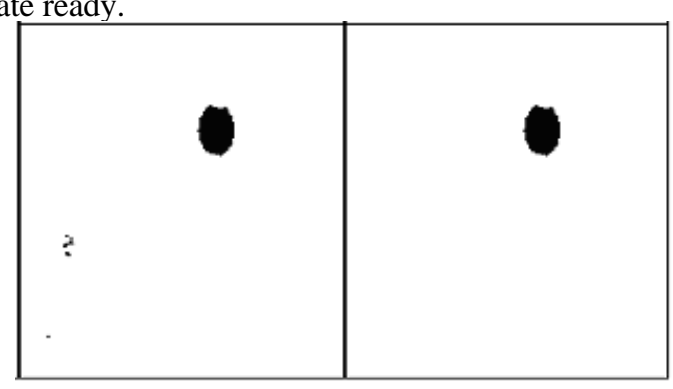

Figure 4. image comparison before and after

\section{Image area mark and small area to eliminate}

Recursive algorithm is a kind of binary image algorithm, but it in serial processor on the computational efficiency is very low, the main reason lies in the need to do image recursive scanning, therefore, the algorithm is mainly applied to parallel machine, and in general robot vision rarely used.

Algorithm is as follows:

- Scanning images, find no mark 1 point (namely image pixel value is 1 point), allocate it a new mark $\mathrm{L}$;

- Recursive distribution mark L to 1 point adjacent point;

- If there is no unlabelled point, then stop ;

- Returns the first step.
Each regional marker, through the calculation area, eliminate small area, figure 5 is for final target image to get the results. The end result of the target image processing.

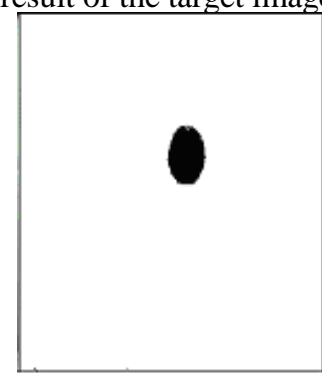

Figure 5. Target image processing results

\section{ENDING}

This paper the robot vision system can finish target recognition on a particular color in the illumination condition change accurately, but also has the very good rapidity and robustness, will be able to accomplish the performance requirements of mobile robot.

\section{REFERENCES}

[1] Zhang Yujin. Image segmentation [M]. Beijing: Science Press, 2001.

[2] ACTS, Active Media Color Tracking Software User Manual version $1[\mathrm{~J}]$, February 2000.

[3] Lu Zongqi.C/C++ image processing programming [M]. Beijing: Qinghua University press, 2003.

[4] Yang Shuying, the second edition of the Visual C++ image processing program design [M]. Beijing: Qinghua University press, 2003.

[5] Jia Yun. Machine vision [M]. Beijing: Science Press, 2000. 